apuntesuniversitarios.upeu.edu.pe

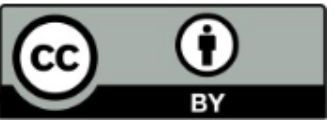

Apuntes Universitarios, 2020: 10(3), julio-setiembre

ISSN: 2304-0335 DOI: https://doi.org/10.17162/au.v10i3.476

\title{
Utilidad percibida de los mapas conceptuales por estudiantes de educación superior
}

\author{
Perceived usefulness of conceptual maps by higher education students \\ Edith Chambi Mescco ${ }^{1 \mathrm{a}}$ \\ Universidad Nacional Mayor de San Marcos ${ }^{1}$ \\ (iD https $/ /$ orcid.org/0000-0002-0535-5906 ${ }^{1}$
}

Recibido: 12 de enero 2020

Aceptado: 01 de junio 2020

\begin{abstract}
Resumen
El presente estudio tuvo como propósito identificar la utilidad de los mapas conceptuales que perciben los estudiantes de primer ciclo de estudios generales de una universidad pública peruana. Los estudiantes elaboraron diversos mapas conceptuales para representar su comprensión conceptual a través del programa Cmap Tools. La metodología de este estudio corresponde al enfoque cuantitativo, por lo que se aplicó el Concept Mapping Questionnaire (CMQ), en su versión en español, a 70 estudiantes matriculados en el periodo académico 2019-I. Los resultados muestran que la mayor parte de los estudiantes consideran que los mapas conceptuales son útiles en alguna y gran medida, principalmente porque les ha ayudado a aclarar las interrelaciones que hay entre los contenidos de la asignatura, a pensar de forma independiente $\mathrm{y}$ a comprender mejor sus aprendizajes.
\end{abstract}

Palabras clave: mapas conceptuales, aprendizaje significativo, educación superior, Cmap Tools, estrategia de aprendizaje.

\begin{abstract}
The purpose of the present work was to identify the usefulness of the conceptual maps that the first cycle students of general studies of a Peruvian public university perceive. Students produced various concept maps to represent their conceptual understanding through the Cmap Tools program. The methodology of this study corresponds to the quantitative approach, so the Concept Mapping Questionnaire (CMQ), in its Spanish version, was applied to 70 students enrolled in the 2019-I academic period. The results show that most of the students consider that concept maps are
\end{abstract}

${ }^{\mathrm{a}}$ Correspondencia al autor:

E-mail: katechambi@gmail.com 
useful to some extent, mainly because it has helped them to clarify the interrelationships between the contents of the subject, to think independently and to better understand their learnings.

Keywords: Concept maps, meaningful learning, higher education, Cmap Tools, learning strategy

\section{Introducción}

Los estudiantes que ingresan a la vida universitaria necesitan ser capaces de aprender autónomamente para que puedan desenvolverse de forma idónea durante sus estudios de educación superior y más aún durante toda su vida, puesto que además del desarrollo de las competencias profesionales, necesitan desarrollar las genéricas que se potencian desde los Estudios Generales. Una de las estrategias didácticas que los docentes han empleado desde hace varias décadas es el mapa conceptual, como herramienta de enseñanza-aprendizaje centrada en el estudiante con el fin de lograr los aprendizajes esperados.

Los mapas conceptuales fueron desarrollados hacia el año 1972 durante el transcurso del programa de investigación de Joseph D. Novak. Su propuesta de uso, como estrategia de aprendizaje, se basa en la teoría del aprendizaje significativo de David Ausubel (Campos, 2005; Rovira, 2016). Se trata de una proyección práctica de la teoría del aprendizaje de Ausubel, al ser una técnica que no busca la repetición memorística de la información, sino el aprendizaje significativo (Ontoria et al., 2011), pues al aprender de memoria no se hace ningún esfuerzo para relacionar las nuevas ideas con las ya existentes y relevantes que forman parte de la estructura cognitiva (Novak, 2010), lo que sí se lograría durante la elaboración de los mapas conceptuales.

Ausubel sostiene que las estructuras cognitivas se refieren al conocimiento y organización de un tema; estas estructuras deciden la significación de las ideas nuevas para su incorporación y retención (Ontoria et al., 2011). Por lo que el aprendizaje significativo implica una conexión entre el conocimiento nuevo y el ya existente en la estructura cognitiva del individuo, pero no se trata de una asociación arbitraria, sino de una basada en la intención del estudiante, siendo así el aprendizaje un proceso activo y personal (Ausubel, Novak y Hanesian, 1989; Daley y Torre, 2010). De ahí la importancia de la motivación que tenga el estudiante en su proceso de aprendizaje, pues aquella será un factor que influya en la interacción genuina del nuevo conocimiento y el que ya existe; el mapa conceptual se convierte en un medio para lograrlo, pues le da la oportunidad al estudiante de tener un rol activo al ser él quien lo construya de forma consciente. 
Por lo que se refiere a los mapas conceptuales, estos se definen como herramientas gráficas que se emplean para organizar y representar el conocimiento (Novak y Cañas, 2006). Se usan para vincular una serie de conceptos pertenecientes a un tema en particular. Uno de sus elementos es el concepto, que se define como una regularidad percibida en eventos u objetos, o registros de eventos u objetos designados por una etiqueta, la cual, generalmente, es una palabra (Novak y Cañas, 2006); además, en el mapa conceptual, los conceptos se relacionan gracias al uso de las palabras enlace, lo que a su vez permite la formación de proposiciones que son unidades semánticas (Novak y Gowin, 2002). Por tanto, en su elaboración se tendría que considerar la selección de conceptos de un dominio del conocimiento en particular y las palabras enlace más apropiadas que permitan construir proposiciones o afirmaciones coherentes que den cuenta de la comprensión cabal de cada concepto organizado por el estudiante en el mapa.

Esta herramienta gráfica también se caracteriza por representar los conceptos de forma jerárquica, desde los más inclusivos y generales hasta los más específicos (Ontoria et al., 2011). Asimismo, en el mapa conceptual se permite la inclusión de los enlaces cruzados, estos tienen la función de vincular los conceptos de diferentes segmentos del mapa conceptual, su utilidad es que ver cómo un concepto, en un dominio de conocimiento representado en el mapa, está relacionado a un concepto de otro dominio (Novak y Cañas, 2006). Por otro lado, los ejemplos pueden también ser incorporados en los mapas conceptuales, puesto que ayudan a aclarar el significado de un concepto (Novak y Cañas, 2006). El impacto visual es también otro componente básico, para lograrlo se recomienda que los conceptos se presenten encerrados en figuras geométricas, la más utilizada es la elipse (Acosta y Acosta, 2010).

Una de las ventajas del uso del mapa conceptual es que, durante su elaboración, el individuo representa su comprensión de los conceptos y las relaciones existentes entre estos, relaciones ya conocidas; sin embargo, hay que considerar que también el estudiante tiene la oportunidad de desarrollar nuevas relaciones conceptuales, por lo que la elaboración de estos mapas promueve la creatividad (Novak y Gowin, 2002); además de ser efectivo en la mejora de las habilidades de pensamiento crítico (Daley y Torre, 2010; Moattari et al., 2014).

Asimismo, el uso de los mapas conceptuales permite el desarrollo no solo de la parte intelectual de individuo, sino también de la personal, por el papel central que le otorga al estudiante, la atención a sus aportaciones incrementa su autoestima (Ontoria et al., 2011), es vital reconocer 
que puede ser una herramienta que incremente la motivación en los estudiantes al darles la posibilidad de que confien más en sí mismos al tomar consciencia de sus logros.

Los mapas conceptuales pueden ser utilizados no solo como una herramienta para apoyar el aprendizaje en la organización y presentación de la información, sino también como una herramienta para la evaluación del aprendizaje (Martínez, 2012). Los docentes tienen la posibilidad de recoger información sobre los logros esperados a través de los mapas conceptuales que elaboran sus estudiantes, lo cual servirá para brindar la retroalimentación y tomar decisiones con respecto a sus prácticas docentes.

La forma de presentar el mapa conceptual y su tamaño podrían variar dependiendo de su audiencia y propósito, este último puede ser: evaluación de los estudiantes, obtención y archivo de información, intercambio de conocimientos, organizadores de la información, presentaciones orales y uso personal como para tomar notas o aprender un tema nuevo, entre otros usos (Cañas, Reiska y Novak, 2016). Además, existen diversos programas para la elaboración de mapas conceptuales, uno de ellos es Cmap Tools desarrollado en el Instituto de Cognición Humana y Mecánica en Florida. Este software brinda la posibilidad de construir, guardar y modificar mapas conceptuales para enriquecer el contenido digital del mismo, incluso permite a los usuarios colaborar a distancia en la construcción de sus mapas y publicarlos para que cualquiera pueda acceder a ellos (Pontes, Serrano y Muñoz, 2015); lo que permite la posibilidad de una construcción fácil de los mapas (Novak, 2010; Ferreira, Cohrs, y De Domenico, 2012). Hay que resaltar que la elaboración de mapas con la ayuda de un software, aporta en la oportunidad que tienen los estudiantes de modificar sus mapas con facilidad, por ejemplo, luego de revisar información adicional, de participar en otras experiencias educativas o recibir la retroalimentación del docente o de sus pares.

Se han realizado diversos estudios en torno a la eficacia de los mapas conceptuales para el desarrollo de los aprendizajes en educación superior. Uno de ellos es el de Hernández y Avilés (2019) quienes evaluaron las potencialidades de los mapas conceptuales como herramienta de sistematización de conocimientos en la autopreparación de los estudiantes en la asignatura de análisis químico; para este fin los estudiantes elaboraron mapas conceptuales sobre diversos contenidos. A pesar de los errores que se identificaron en los mapas elaborados, los resultados revelaron que los estudiantes reconocen las potencialidades del mapa conceptual para facilitar la 
comprensión de los contenidos de la asignatura, los autores sugieren que se cuente con un curso de formación pedagógica que ayude a los estudiantes a utilizar este tipo de mapa. La preocupación de estudios como este ha girado en torno a la satisfacción estudiantil, lo cual es importante debido a que, como ya se indicó, la intención del estudiante es determinante para el logro de sus aprendizajes.

En resumen, los mapas conceptuales tienen diversas ventajas según los estudios que se han realizado; sin embargo, habría que saber en qué medida los estudiantes se encuentran satisfechos con el uso de esta herramienta. Si bien hay varias investigaciones en torno a esta necesidad, no hay una para la población descrita en este estudio, por lo que el objetivo del mismo fue identificar los usos que perciben los estudiantes de primer ciclo acerca del mapa conceptual.

\section{Método}

Se realizó un estudio descriptivo y cuantitativo a través del cual se aplicó el Concept Mapping Questionnaire (CMQ), en su versión original obtuvo un índice de 0,92 en el alfa de Cronbach (Buldu y Buldu, 2010); asimismo, la versión en español del CMQ pasó también por un proceso de confiabilidad y se obtuvo 0,95 en el alfa de Cronbach (Domínguez y Vega, 2018). El cuestionario consta de una escala tipo Likert (Nada: 1, Muy poco: 2, En alguna medida: 3 y En gran medida: 4) y doce ítems. Debido a cuestiones de factibilidad, se empleó el muestreo por conveniencia (Otzen y Manterola, 2017). La muestra estuvo conformada por 70 estudiantes de primer ciclo del semestre académico 2019-1 de la Escuela de Estudios Generales de una universidad pública peruana. Los resultados fueron organizados y analizados de forma descriptiva mediante el software SPSS 25.

\section{Resultados}

Los datos se obtuvieron tras la aplicación del CMQ en su versión en español a los estudiantes de la muestra estudiada, quienes durante el semestre académico 2019-I elaboraron mapas conceptuales de los temas propios de la misma asignatura; cabe resaltar que la elaboración se realizó usando el programa Cmap Tools. Los mapas fueron evaluados y retroalimentados por el docente, quien previamente brindó orientaciones sobre el proceso de su elaboración. Sobre las características de la muestra, la media de las edades de los estudiantes fue de 18,8. Asimismo, de acuerdo al género, el 50\% (35) de la muestra corresponde al género femenino y el otro 50\% (35), 
al masculino. Los porcentajes en torno a los usos percibidos por los estudiantes con respecto al mapa conceptual se presentan en la tabla 1, mientras que los promedios de los puntajes obtenidos por ítem se encuentran en la tabla 2 .

\section{Tabla 1}

Percepciones de los estudiantes sobre el uso de los mapas conceptuales.

\begin{tabular}{|c|c|c|c|c|c|}
\hline N.o & Declaraciones & Nada & $\begin{array}{l}\text { Muy } \\
\text { Poco }\end{array}$ & $\begin{array}{c}\text { En } \\
\text { alguna } \\
\text { medida }\end{array}$ & \begin{tabular}{|} 
En gran \\
medida
\end{tabular} \\
\hline 1 & $\begin{array}{l}\text { Me han ayudado a comprender los conceptos clave sobre } \\
\text { los temas de clase. }\end{array}$ & 0 & 0 & 57,1 & 42,9 \\
\hline 2 & $\begin{array}{l}\text { Me han ayudado a mejorar mi aprendizaje sobre los } \\
\text { contenidos del curso. }\end{array}$ & 0 & 2,9 & 62,9 & 34,3 \\
\hline 3 & $\begin{array}{l}\text { Han aumentado mi motivación hacia el contenido del } \\
\text { curso. }\end{array}$ & 1,4 & 12,9 & 61,4 & 24,3 \\
\hline 4 & Me han ayudado a aumentar mi participación en la clase. & 4,3 & 11,4 & 52,9 & 31,4 \\
\hline 5 & $\begin{array}{l}\text { Me han ayudado a comunicar a otros mi aprendizaje en } \\
\text { la clase. }\end{array}$ & 0 & 14,3 & 61,4 & 24,3 \\
\hline 6 & Me han estimulado a pensar de forma independiente. & 0 & 12,9 & 42,9 & 44,3 \\
\hline 7 & $\begin{array}{l}\text { Me han ayudado a aprender cooperativamente con otras } \\
\text { personas en la clase. }\end{array}$ & 5,7 & 11,4 & 52,9 & 30 \\
\hline 8 & $\begin{array}{l}\text { Me han permitido realizar conexiones entre conceptos } \\
\text { que han desafiado mi pensamiento. }\end{array}$ & 0 & 2,9 & 54,3 & 42,9 \\
\hline 9 & $\begin{array}{l}\text { Me han ayudado a aclarar las interrelaciones entre los } \\
\text { contenidos del curso. }\end{array}$ & 0 & 5,7 & 40 & 54,3 \\
\hline 10 & $\begin{array}{l}\text { Me han ayudado a ver los componentes que faltan en mi } \\
\text { aprendizaje sobre el contenido del curso. }\end{array}$ & 0 & 8,6 & 50 & 41,4 \\
\hline 11 & $\begin{array}{l}\text { Me han ayudado a visualizar algunos conceptos } \\
\text { expresados ambiguamente por el profesor. }\end{array}$ & 0 & 8,6 & 60 & 31,4 \\
\hline 12 & $\begin{array}{l}\text { Me han ayudado a comprender mejor mi aprendizaje en } \\
\text { la clase. }\end{array}$ & 0 & 5,7 & 38,6 & 55,7 \\
\hline
\end{tabular}

Fuente: Elaboración propia 
Tabla 2

Medias de puntajes obtenidos por ítem.

\begin{tabular}{|c|c|c|c|c|c|}
\hline Ítems & $\mathrm{N}$ & Mínimo & Máximo & Media & $\begin{array}{c}\text { Desv. } \\
\text { Desviación }\end{array}$ \\
\hline $\begin{array}{l}\text { Me han ayudado a comprender los } \\
\text { conceptos clave sobre los temas de } \\
\text { clase. }\end{array}$ & 70 & 3 & 4 & 3,43 & ,498 \\
\hline $\begin{array}{l}\text { Me han ayudado a mejorar mi } \\
\text { aprendizaje sobre los contenidos del } \\
\text { curso. }\end{array}$ & 70 & 2 & 4 & 3,31 &, 526 \\
\hline $\begin{array}{l}\text { Han aumentado mi motivación hacia } \\
\text { el contenido del curso. }\end{array}$ & 70 & 1 & 4 & 3,09 & 654 \\
\hline $\begin{array}{l}\text { Me han ayudado a aumentar mi } \\
\text { participación en la clase. }\end{array}$ & 70 & 1 & 4 & 3,11 & ,772 \\
\hline $\begin{array}{l}\text { Me han ayudado a comunicar a otros } \\
\text { mi aprendizaje en la clase. }\end{array}$ & 70 & 2 & 4 & 3,10 & 617 \\
\hline $\begin{array}{l}\text { Me han estimulado a pensar de } \\
\text { forma independiente. }\end{array}$ & 70 & 2 & 4 & 3,31 & ,692 \\
\hline $\begin{array}{llr}\text { Me han ayudado a } & \text { aprender } \\
\text { cooperativamente } & \text { con } & \text { otras } \\
\text { personas en la clase. } & & \\
\end{array}$ & 70 & 1 & 4 & 3,07 & ,804 \\
\hline $\begin{array}{l}\text { Me han permitido realizar } \\
\text { conexiones entre conceptos que han } \\
\text { desafiado mi pensamiento. }\end{array}$ & 70 & 2 & 4 & 3,40 & ,549 \\
\hline $\begin{array}{l}\text { Me han ayudado a aclarar las } \\
\text { interrelaciones entre los contenidos } \\
\text { del curso. }\end{array}$ & 70 & 2 & 4 & 3,49 & ,608 \\
\hline $\begin{array}{l}\text { Me han ayudado a ver los } \\
\text { componentes que faltan en } \\
\text { aprendizaje sobre el contenido del } \\
\text { curso. }\end{array}$ & 70 & 2 & 4 & 3,33 & ,631 \\
\hline 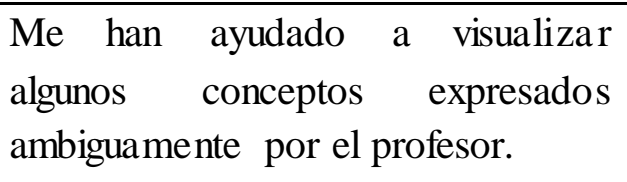 & 70 & 2 & 4 & 3,23 & ,594 \\
\hline $\begin{array}{l}\text { Me han ayudado a comprender } \\
\text { mejor mi aprendizaje en la clase. }\end{array}$ & 70 & 2 & 4 & 3,50 & ,608 \\
\hline
\end{tabular}

Fuente: Elaboración propia 
$\mathrm{El}$ análisis muestra que los estudiantes perciben mayor grado de satisfacción sobre el uso de los mapas conceptuales en los ítems 6,9 y 12. Se analizaron también las puntuaciones que se obtuvieron por cada estudiante de acuerdo a la escala, las mismas que varían entre 27 y 48 . La media de tales puntuaciones que resulta es 39,37 y la desviación estándar es 4,09. Esto significa que los estudiantes consideran el uso de los mapas conceptuales como útil en alguna medida al ser la media de las puntuaciones 3,28 (39.37/12).

\section{Discusión}

Los resultados mostraron que, en su mayoría, los estudiantes consideran los mapas conceptuales como útiles en gran medida (nivel 4 en la escala) en tres aspectos: logra estimularlos a pensar de forma independiente, ayuda a aclarar las interrelaciones que hay entre los contenidos del curso y a comprender mejor su aprendizaje en la clase. Como ya las bases teóricas lo indican, los mapas conceptuales resultan de utilidad para relacionar los conceptos e integrarlos, con el fin de comprender los contenidos, siendo el aprendizaje responsabilidad del individuo (Novak y Gowin, 2002), utilidad que fue reconocida por la mayor parte de los estudiantes que participaron en el estudio.

Se evidencia también en la tabla 1 que los estudiantes consideran útiles los mapas conceptuales en alguna medida (nivel 3 en la escala) con un porcentaje $\geq 60$ en los ítems referidos a "Me han ayudado a mejorar mi aprendizaje sobre los contenidos del curso", "Han aumentado mi motivación hacia el contenido del curso" y "Me han ayudado a visualizar algunos conceptos expresados ambiguamente por el profesor". Los mapas conceptuales no solo permiten que el estudiante genere ideas e integre los nuevos conocimientos a los que ya posee, sino también permite que evalúe y mida la comprensión de conceptos (Arellano y Santoyo, 2009), que pueda identificar errores (Mukherjee, Cabrera y Silva, 2018), puesto que el aprendizaje significativo no implica la ausencia de errores conceptuales como utilizar conceptos erróneos, concepciones alternativas, nociones ingenuas y nociones precientíficas (Correia y Cicuto, 2014). Todo ello contribuye a que se sientan más seguros al utilizar mapas conceptuales, pues se incrementa su interés por el aprendizaje (Maldonado y Romero, 2016). Un buen porcentaje de estudiantes percibe esta utilidad, que se evidencia en las oportunidades que tuvieron de sentirse más motivados al aplicar una estrategia centrada en ellos; y, a su vez, poder aclarar conceptos y corregir las ideas erróneas que 
pudieron tener sobre los mismos, lo cual en cierta medida les permitió tomar conciencia de cuánto estaban aprendiendo.

Es necesario señalar que los porcentajes más altos corresponden al nivel 3 de la escala, estos resultados coinciden con un estudio realizado en Colombia (Domínguez y Vega, 2018) en el que se aplicó el mismo instrumento (CMQ), los estudiantes de la muestra estudiada opinan que los mapas conceptuales son estrategias útiles en cierta medida para la síntesis de información, puesto que la media de las puntuaciones en el postest resultó entre 3,38 y 3,49. Los resultados también coinciden con los hallazgos de un estudio en el cual se midieron las percepciones de los estudiantes sobre el uso de los mapas conceptuales con el uso del CMQ, del cual resultó que la media de las puntuaciones fue 3,33 lo que corresponde a la escala "en alguna medida" (Buldu y Buldu, 2010). Igualmente, en un estudio cualitativo realizado con estudiantes de Enfermería, se concluye que la percepción sobre los mapas conceptuales es favorable, debido a que permiten la asociación de conceptos y con ello la comprensión de los temas al unir las brechas entre lo que desconocían y el nuevo conocimiento, corrigiendo errores, lo cual favorece el aprendizaje significativo (Rahnama y Mardani-Hamooleh, 2017). En otro estudio, se recibió una respuesta muy positiva sobre la utilidad de los mapas conceptuales para memorizar, aumentar la confianza y comprender el tema (Joshi y Vyas, 2018).

Asimismo, es necesario señalar que, solo en tres de los ítems, la mayoría de los estudiantes percibieron la utilidad de los mapas conceptuales en gran medida. Posiblemente la dificultad que demanda expresar su comprensión del tema en el mapa (Cañas, Reiska y Novak, 2016) sea un factor que influya en este resultado. Es un desafío para el docente la preparación de instrucciones claras, además de capacitar y alentar adecuadamente a los estudiantes sobre el uso del mapa conceptual (Jaafarpour, Aazami, y Mozafari, 2016), pues los estudiantes generalmente no están familiarizados con esta herramienta gráfica (Carr-Lopez et al., 2014). Quizás puedan no entender cómo construirlos, por lo que se puede producir resistencia a emplearlos, la cual también suele estar relacionada al tiempo mayor que lleva elaborar este tipo de mapa (Daley y Torre, 2010; Jaafarpour, Aazami, y Mozafari, 2016). Frente a esta situación, se requiere que el docente dedique tiempo adicional en las sesiones para asegurar que los estudiantes tengan dominada la habilidad de crear mapas conceptuales (Kostromina y Gnedykh, 2016). El compromiso que muestren los docentes y los gestores de la institución para la capacitación en elaboración de mapas conceptuales, 
dedicar tiempo a la preparación de los estudiantes tanto en la forma apropiada de construir los mapas, como en el manejo del software; así como la incorporación de sesiones dedicadas a este objetivo en el silabo de la asignatura; será indispensable para el logro de los aprendizajes esperados a través de esta estrategia.

Por todo lo expuesto, hacen falta más estudios para determinar qué otras dificultades presentaron los estudiantes durante la aplicación de los mapas conceptuales y en qué medida contribuyó al logro de los aprendizajes de la asignatura. Por otro lado, si bien, este estudio ha logrado cumplir con su objetivo de identificar las percepciones de los estudiantes; por el tipo de muestreo empleado, los resultados obtenidos no pueden generalizarse. Se espera que este estudio sea el inicio de otros que puedan cubrir esta necesidad.

\section{Conclusión}

El mapa conceptual es una estrategia de enseñanza-aprendizaje centrada en el estudiante que favorece el logro de aprendizajes significativos al cumplir la función de representar la comprensión conceptual de los estudiantes en torno a algún campo del conocimiento. Esta investigación ha puesto de manifiesto que las percepciones de los estudiantes sobre el uso de los mapas conceptuales son positivas, puesto que consideran que son útiles en alguna y gran medida, principalmente porque les ha ayudado a aclarar las interrelaciones que hay entre los contenidos del curso, a pensar de forma independiente y a comprender mejor sus aprendizajes.

\section{Referencias}

Acosta, S., y Acosta, R. (2010). Los mapas conceptuales y su efecto en el aprendizaje del conocimiento biológico. Omnia, 2(16), 209-225. Recuperado de: https://www.redalyc.org/pdf/737/73715084012.pdf

Arellano, J., y Santoyo, M. (2009). Investigar con mapas conceptuales. Procesos Metodológicos. Madrid: Narcea Ediciones.

Ausubel, D., Novak, J., y Hanesian, H. (1989). Psicología educativa. México: Trillas.

Buldu, M., y Buldu, N. (2010). Concept mapping as a formative assessment in college classrooms: Measuring usefulness and student satisfaction. Procedia Social and Behavioral Sciences 2 (2), 2099-2104. Doi: 10.1016/j.sbspro.2010.03.288 
Campos, A. (2005). Mapas conceptuales, mapas mentales y otras formas de representación del conocimiento. Bogotá: Cooperativa Editorial Magisterio.

Cañas, A., Reiska, P., y Novak, J. (2016). Is My Concept Map Large Enough? In: Cañas A., Reiska P., Novak J. (eds) Innovating with Concept Mapping. CMC 2016. Communications in Computer and Information Science, Springer, 635, 128-143. Recuperado de: https://doi.org/10.1007/978-3-319-45501-3_10

Carr-Lopez, S., Galal, S., Vyas, D., Patel, R., y Gnesa, E. (2014). The Utility of Concept Maps to Facilitate Higher-Level Learning in a Large Classroom Setting. American Journal of Pharmaceutical Education, 78(9), 170. doi: 10.5688/ajpe789170

Correia, P., y Cicuto, C., (2014). Neighbourhood Analysis to Foster Meaningful Learning Using Concept Mapping in Science Education, Science Education International Journal, 25(3) 259-282. https://eric.ed.gov/?q=conceptual+map yff $1=$ subHigher+Educationyff $2=$ =eduHigher+Educati onyff $3=$ subConcept + Mappingyid=EJ1044456

Daley, B., y Torre, D. (2010). Concept maps in medical education: an analytical literature review. Medical Education 44(5): 440-448. doi:10.1111/j.1365-2923.2010.03628.

Domínguez, L., y Vega, N. (2018). Efectos del mapa conceptual sobre la síntesis de informac ión en un ambiente de aprendizaje interactivo: Un estudio preexperimental. Educación Médica, 408. Recuperado de: https://doi.org/10.1016/j.edumed.2018.08.00

Ferreira, P., Cohrs, C., y De Domenico, E. (2012). Software CMAP TOOLS TM to build concept maps: an evaluation by nursing students. Revista Da Escola de Enfermagem Da U S P, 46(4), 967-972. Recuperado de: https://doi.org/10.1590/s0080-62342012000400026

Hernández, A., y Aviles, E. (2019). El empleo de los mapas conceptuales como herramienta del estudiante para sistematizar los conocimientos. Trasformación,15(2), 49-65. Recuperado de: http://scielo.sld.cu/scielo.php?script=sci_abstractypid=S2077-29552019000200049

Jaafarpour, M., Aazami, S., y Mozafari, M. (2016). Does concept mapping enhance learning outcome of nursing students? Nurse Education Today, 36, 129-132. Recuperado de: https://doi.org/10.1016/j.nedt.2015.08.029

Joshi, U., y Vyas, S. (2018). Assessment of perception and effectiveness of concept mapping in learning epidemiology. Indian Journal of Community Medicine, 43(1), 37. Recuperado de: https://doi.org/10.4103/ijcm.IJCM_375_16

Kostromina, S., y Gnedykh, D. (2016). Russian Experience in Application of Concept Maps in Education. Communications in Computer and Information Science, Springer, 635, 159-168. Recuperado de: https://doi.org/10.1007/978-3-319-45501-3_12 
Maldonado, I., y Romero, L. (2016). Eficacia de los mapas conceptuales en el desarrollo de las competencias en información electrónica. Anales de Documentación, 19(1), 1-18. Recuperado de: https://doi.org/10.6018/analesdoc.19.1.231871

Martínez, L. (2012). Using an improved virtual learning environment for engineering students. European Journal of Engineering Education, 37(3), 229-241. Recuperado de: https://doi.org/10.1080/03043797.2012.678985

Moattari, M., Soleimani, S., Moghaddam, N. J., y Mehbodi, F. (2014). Clinical concept mapping: Does it improve discipline-based critical thinking of nursing students? Iranian Journal of Nursing and Midwifery Research, 19(1), 70-76. Recuperado de: http://www.ncbi.nlm. nih.gov/pubmed/24554963

Mukherjee, S., Cabrera, A., y Silva, M. (2018). Evaluation of group concept mapping during advanced pharmacy practice experiences. Currents in Pharmacy Teaching and Learning, 10(12), 1616-1623. Recuperado de: https//doi.org/10.1016/j.cptl.2018.09.009

Novak, J., y Cañas, A. (2006). The Theory Underlying Concept Maps and How to Construct Them. Florida Institute for Human and Machine Cognition. Recuperado de: https://cmap.ihmc.us/publications/researchpapers/theoryc maps/TheoryUnderlyingConcept Maps.bck-11-01-06.htm

Novak, J., y Gowin, B. (2002). Aprendiendo a aprender. Barcelona: Ediciones Martínez Roca.

Novak, J. (2010). Learning, Creating, and Using Knowledge: Concept maps as facilitative tools in schools and corporations. Journal of e-Learning and Knowledge Society 6(3), 21-30. Recuperado de: https://www.learntechlib.org/p/43512/

Ontoria A., Ballesteros A., Cuevas, C., Giraldo, L., Martin, L., Molina, A., Rodríguez, A., y Velez, U. (2011). Mapas conceptuales una técnica para aprender. Madrid: Narcea Ediciones.

Otzen, T., y Manterola, C. (2017). Técnicas de Muestreo sobre una Población a Estudio. Morphol, 35(1), 227-332. Recuperado de: https://scielo.conicyt.cl/pdf/ijmorphol/v35n1/art37.pdf

Pontes, A., Serrano, R., y Muñoz, J. (2015). Los mapas conceptuales como recurso de interés para la formación inicial del profesorado de enseñanza secundaria: Opiniones del alumnado de ciencias sociales y humanidades. Educación XX1, 18(1), 99-124. doi:10.5944/educXX1.18.1.12313

Rahnama, F., y Mardani-Hamooleh, M. (2017). Iranian nursing students' perceptions regarding use of concept mapping: a content analysis. Res Dev Med Educ, 6(1), 45-50. doi: 10.15171/rdme.2017.008

Rovira, C. (2016). Theoretical foundation and literature review of the study of concept maps using eye tracking methodology. El profesional de la información, 25(1), 59-73. Recuperado de: http://www.elprofesionaldelainformacion.com/contenidos/2016/ene/07.pdf 